\title{
Improving High Availability and Reliability of Health Interoperability Systems
}

\author{
Fernando Marins ${ }^{1}$, Luciana Cardoso ${ }^{1}$, Filipe Portela ${ }^{2}$, Manuel Santos ${ }^{2}$, \\ António Abelha ${ }^{1}$, and José Machado ${ }^{1}$ * \\ ${ }^{1}$ University of Minho, CCTC, Department of Informatics, Braga, Portugal, \\ ${ }^{2}$ University of Minho, Algoritmi, Department of Information Systems, Guimarães, \\ Portugal \\ \{f .abreu.marins, lucianacardoso05\}@gmail.com \\ \{cfp, mfs\}@dsi.uminho.pt \\ \{abelha, jmac\}@di.uminho.pt
}

\begin{abstract}
The accessibility and availability of patient clinical information are a constant need. The Agency for Interoperation, Diffusion and Archive of Medical Information (AIDA) was then developed to ensure the interoperability among healthcare information systems successfully. AIDA has demonstrated over time the need for greater control over its agents and their activities as the need for monitoring and preventing its machines and agents.

This paper presents monitoring and prevention systems that were developed for machines and agents, which allow not only prevent faults, but also watch and evaluate the behaviour of these components through monitoring dashboards. The Biomedical Multiagent Platform for Interoperability (BMaPI) implemented in Centro Hospitalar do Porto (CHP) revealed provide the necessary data and functionalities capable to manage and to monitor agents' activities. It was found that the prevention systems identified critical situations successfully, contributing to an increase in the integrity and availability of AIDA implemented in CHP.
\end{abstract}

Keywords: Interoperability, Health Information Systems, Monitoring System, Fault Forecasting.

\section{Introduction}

The health information systems (HIS) have been a topic increasingly attractive in the field of scientific research, since provide to health professionals a better contact with the equipment, thus reducing the number and incidence of medical errors. Also contribute to a reduction of costs and may provide a means to improve the management hospital. Due to its specificity, the HIS constitutes a complex environment of heterogeneous systems, distributed and ubiquitous, communicating in different ways, integrating specific medical equipment that are

\footnotetext{
* Corresponding Author
} 
developed by different entities, with different goals. Thus, increasingly emerges the need of HIS stay in constant communication $[1,2]$.

The possibility and the need to communicate is one of the main characteristics of human beings. Also HIS needs to communicate and cooperate in order to improve their overall performance, their usefulness, the quality of diagnoses, but mainly to improve the quality of patient care. Cooperation and exchange of data and information is in fact one of the most important characteristics, and essence for the optimization of existing resources and improving the decision making process through consolidation, verification and dissemination of information [3].

It is presented then the concept of interoperability, which defines the capacity for independent systems to exchange meaningful information and perform actions belonging to other systems in order to operate together towards a mutual goal $[3,4]$.

In order to aggregate and consolidate all relevant information, a solid and efficient process of integration and interoperability has to be developed. This process must take into account the scalability, flexibility, portability and security (confidentiality, integrity and availability) when applied in this environment. The Agency for Interoperation, Diffusion and Archive of Medical Information (AIDA) arises to be part of this process of integration of different sources of information through the use of different protocols and methodologies. Furthermore this platform also provides tools to implement and facilitate the communication with humans through web based services [1]. However, this platform contains some limitations of which highlights the difficulty in controlling the AIDA's agents and the need to monitor and prevent failures in their components. To address these two limitations and improve the availability and functionality of AIDA, several systems have been developed for monitoring and prevention and they are presented in Section 4. Section 2 presents and evaluates the AIDA platform. Section 3 presents MEWS, a fault forecasting model used in Intensive Care Units, the prevention systems developed are inspired in this model. Finally, Section 5 presents the results about the implementation of these systems and Section 6 presents conclusions and future work.

\section{AIDA}

The techniques based on Artificial Intelligence (AI) has been demonstrated a high potential when introduced into solutions applied to hospital environments. Many of these solutions are already in production in several portuguese healthcare institutions, centred in systems integration and decision support systems. Also in the interoperability area, these techniques have been highlighted, namely a solution developed by a group of artificial intelligence at University of Minho called AIDA [5] is already a central unit for interoperability in several large organizations such as Centro Hospitalar do Porto (CHP), Centro Hospitalar do Tâmega e Sousa, Centro Hospitalar do Alto Ave and Unidade local de Saúde do Norte Alentejano. This agent-based platform has demonstrated a great adaptability, modularity and effectiveness through the use of a basic Multi-Agent 
System (MAS) that has grown according to the particular needs of each institution.

AIDA was designed to assist medical applications in the form of a network of intelligent systems for the information processing, its major subsystems, their functional role, and for control the flow of information with a level of autonomy adjustable. It was developed to allow the diffusion and the integration of information generated in a healthcare environment. It is a complex system composed of specialized subsystems understood as intelligent and flexible entities, in this work designed as intelligent agents, responsible for tasks such as the communication among heterogeneous systems, the sending and the receiving of information (e.g. clinical reports, images, sets of data, prescriptions, etc.), management and storage of information and responding to requests in a timely and correct manner $[5,6]$.

This platform uses many integration resources, namely technologies like Service Oriented Architectures (SOA) and MAS for the implementation of interoperability in a distributed, specific and conform to a standard manner, comprising all the service providers within the healthcare institution. The main objective of this system is, as the name implies, integrate, disseminate and archive large sets of data arising several sources (e.g. services, departments, units, computers and medical equipments, etc.). It also provides tools to implement and facilitate the communication with human beings through web-based services [3]. The AIDA platform has taken a vital role in the normal functioning of the SIH in the institutions where it is implemented. Thus it is very important ensure that it offers the best features and that the users directly related to its operation are satisfied with its performance. For this, were raised the main advantages and disadvantages of the AIDA platform, presented in Table 1.

Table 1. AIDA's advantages and disadvantages.

\begin{tabular}{|l|l|}
\hline \multicolumn{1}{|c|}{ Advantages } & \multicolumn{1}{c|}{ Disadvantages } \\
\hline $\begin{array}{l}\text { High power managing changes in the } \\
\text { system; }\end{array}$ & \\
- Objets's customization ability; & - Need for a great education and train- \\
- High availability, accessibility and & ing of the professionals; \\
timely support; & Physical resources old, slow and lim- \\
- High security of the information and & ited; \\
the system; & - Difficulty in the controlling agents and \\
- Technologically modern; & its activities; \\
- Ease of maintenance and simplicity of & - Necessity of monitoring and prevent- \\
use of the system. & ing failures of AIDA's components. \\
& \\
\hline
\end{tabular}

Although it is possible to verify that the disadvantages of AIDA does not directly influence its operation. This work aims to circumvent and eliminate the last two disadvantages presented in the Table 1 by developing and implementing the BMaPI whose main goal addresses the need for a greater control over the agents and monitoring their pro-activity, as well as by developing and 
implementing monitoring and fault forecasting systems of AIDA's components, particularly its machines and its agents. To achieve the prediction of faults it is crucial to create fault forecasting models. For this project the models created (presented in the Section 4) are based on MEWS.

\section{MEWS}

MEWS stands for Modified Early Warning Score and this model has the purpose of predicting serious health problems. It is used especially in Intensive Care Units and it assumes that a serious health problem is often preceded by physiological deterioration. For the MEWS succeed, it is crucial to resort to monitoring of patient's vital signs. Based on the Table 2, scores are assigned to each parameter. The sum of the scores obtained means the patient's level of risk. In this way, physicians are able to prevent serious problems [7].

Table 2. MEWS Scores (adapted) [7].

\begin{tabular}{|c|c|c|c|c|c|c|c|}
\hline MEWS Score & 3 & 2 & 1 & $\mathbf{0}$ & 1 & 2 & 3 \\
\hline $\begin{array}{l}\text { Temperature } \\
\left({ }^{\circ} \mathrm{C}\right)\end{array}$ & & $<35.0$ & $35.1-36.0$ & $36.1-38.0$ & $38.1-38.5$ & $>38.6$ & \\
\hline $\begin{array}{l}\text { Heart rate } \\
\left(\min ^{-1}\right)\end{array}$ & & $<40$ & $41-50$ & $51-100$ & $101-110$ & $111-130$ & $>131$ \\
\hline $\begin{array}{l}\text { Systolic BP } \\
(\mathrm{mmHg})\end{array}$ & $<70$ & $71-80$ & $81-100$ & $101-199$ & & $>200$ & \\
\hline $\begin{array}{l}\text { Respiratory } \\
\text { rate }\left(\min ^{-1}\right)\end{array}$ & & $<8$ & & $8-14$ & $15-20$ & $21-29$ & $>30$ \\
\hline $\begin{array}{l}\text { Blood } \\
\text { oxygen }(\%)\end{array}$ & $<85$ & $85-89$ & $90-93$ & $>94$ & & & \\
\hline $\begin{array}{l}\text { Urine output } \\
(\mathrm{ml} / \mathrm{kg} / \mathrm{h})\end{array}$ & $\mathrm{Nil}$ & $<0.5$ & & & & & \\
\hline Neurological & & $\begin{array}{c}\text { New } \\
\text { confusion }\end{array}$ & & Alert & $\begin{array}{l}\text { Reacting } \\
\text { to voice }\end{array}$ & $\begin{array}{l}\text { Reacting } \\
\text { to pain }\end{array}$ & Unresponsive \\
\hline
\end{tabular}

In order to categorize the patient's state based on Table 2, the physicians follow several guidelines such as: if any of the parameters have a score equal to two, the patient must be in observation; if the sum of scores being equal to four or there being an increase of two values, the patient requires urgent medical attention; if a patient has a score higher than four, he is at risk of life [7].

The MEWS's implementation presents several advantages: it allows to analyse the patient's historical over time, through the continuous collection and storage of the patient's vital signs; it also allows to set priorities for the interventions to perform; the process of monitoring and observation of the patient is enriched because it provides information about organism's physiological trends; it assists in medical decision making, once it is based on quantitative criteria; it forecasts situations where the patient requires hospitalization in the Intensive Care Unit $[7,8]$.

MEWS is used in hospitals in some countries such as Australia, the UK and Spain. In the case of Portugal, it is implemented in Centro Hospitalar do Barlavento Algarvio and it is used in researches developed in CHP [6,8,9]. Given the MEWS's advantages and applicability, this model proves to be a reliable option to adapt to AIDA's components. 


\section{Intelligent Systems for Monitoring and Preventing}

Today it is vast the number of platforms developed to create agents and to establish their communication. However the monitoring of agents' activity has been a subject rarely addressed yet. With respect to the fault forecasting systems, some similar works have been done such as systems to predict failures in healthcare databases [9]. The monitoring of AIDA's components (such as its agents and machines) it is fundamental to control their activities, furthermore this process enables the administrators to balance the system's resources properly. Through a successful process of monitoring, it is possible to implement fault forecasting systems, in this case based on MEWS model presented in the Section 3, in order to prevent critical situations that might compromise the proper functioning of AIDA and consequently the quality of the healthcare delivery.

\subsection{Monitoring Systems}

In order to overcome one of the drawbacks of AIDA platform, was designed the Biomedical Multi-agent Platform for Interoperability (BMaPI), a system that allows the control and manage of a community of agents, ensuring their survival in a heterogeneous environment. More specifically, a platform that integrates all agents in the environment, regardless of the machine where they perform their activity. Beyond it ensure that all AIDA's agents communicate, it allows the creation of an interoperable environment. To facilitate the control of the agents, it was also developed a graphical user interface integrated into BMaPI for the user to manage in a simple and attractive way, the agents that constitute the system and their activities. In general, and taking into account the needs described above, the BMaPI was designed to be able to: ensure a greater control over the agents that constitute the AIDA; facilitate the user to the creation and registration of new agents locally or remotely; allow the user to enable and disable services through the launch or stop a specific agent; facilitate scheduling and rescheduling the activity of agents; monitoring the activity of agents.

Several agent platforms have been developed with particular attention to the thematic interoperability and compatibility. Accordingly, the reference model FIPA has emerged as a standard for agents oriented programming, which among other specifications have one to standardize the communication between the agents in order to ensure their social skills. This specification, called FIPA-ACL was used for the development of this platform, to ensure the uniformity of the messages exchanged among the agents. In general terms, BMaPI is based on a multi-thread application respecting the specifications of a client/server architecture making use of TCP/IP sockets to exchange the messages between the stakeholders, the agents. The TCP protocol used confers to BMaPI a great reliability because it guarantees that the information is transported and delivered without any data manipulation. The client/server multi-thread architecture and the property that the server has to communicate with multiple clients at the same time, gives to BMaPI large scalability because this network may gradually grow. In BMAPI, agents of various types can be created, thus being considered 
as a system with great adaptability. It is also a system with great portability once its server can run on any machine, adapting automatically to their properties. Its distributed architecture also benefits the BMaPI reducing your processing time and consequently its time of response. Moreover, these technologies confer on BMaPI a high level of interoperability.

Once the BMaPI ensures AIDA's agents monitoring, it emerges the necessity to control the AIDA's machines activity. After all, it is on these machines that the agents execute their tasks. Besides that, the monitoring of these machines enables the system administrators to evaluate their performance and to balance the resources properly, maximizing AIDA's efficiency.

The selection of performance indicators demands a high knowledge about the system wherein it is intended to implement a monitoring system. The selection should be based on the workload that each component is subjected and parameters that are important to achieve the objectives delineated [10].

In this way, a monitoring system for AIDA's machines was developed. This system collects periodically information from several machines about the CPU and memory usage and disk free space. In order to collect this information, it uses the WMI (Windows Management Instrumentation) technology, which contains a powerful set of tools, based on standards, that enables an easy exchange of data. It uses the credentials that are provided to access remotely objects that contains the desired information. This access is made through a simple querybased language: Windows Query Language (WQL) [11].

It was developed monitoring dashboards through a business intelligence tool named Pentaho Community [12]. These dashboards, which have a friendly graphical interface, allow system administrators to control the machines activities and aids them in making decisions relative to AIDA's resources management.

Once the monitoring process is achieved, it emerges the possibility to implement preventing systems that increases the high availability of AIDA.

\subsection{Preventing Systems}

It was developed two fault forecasting systems: one of the AIDA's machines and another of the AIDA's agents. For the machines, once selected the performance indicators and the monitoring process is in progress, the next step is to adapt MEWS to the AIDA's machines context. The Table 3 presents the score table for the machines. Initially there was an attempt to create a score table based on percentiles, more concretely inspired on the 95-percentile method used for billing in Internet Service Providers and websites [13]. However, the quantifying of the performance indicators by percentiles did not succeed because a reasonable number of warnings false positives were sent per day. The limits for a good performance of a machine varies a lot according to the objectives that the system administrators delineate. In this way, the solution was to create the Table 3 based on default fixed limits that can be modified anytime through an administrative page, for each parameter either generally or specifically for one machine. The limits established as default were discussed among the system administrators, which have a high experience and knowledge about AIDA. 
Table 3. Score table for the machines.

\begin{tabular}{|l|c|c|c|c|}
\hline Score & $\mathbf{0}$ & $\mathbf{1}$ & $\mathbf{2}$ & $\mathbf{3}$ \\
\hline Free processor (\%) & $\geq 50$ & ] $50,25]$ & ] $25,10]$ & $<10$ \\
\hline Free memory (\%) & $\geq 15$ & ] $15,10]$ & ] $10,5]$ & $<5$ \\
\hline Free disk's space (\%) & $\geq 15$ & ] $15,10]$ & ] $10,5]$ & $<5$ \\
\hline
\end{tabular}

As in MEWS, if the sum of the scores is equal to four or there is an increase of two values, it is considered that the situation is grave and a warning on the monitoring dashboard will appear. If the sum of all parameters' score is more than four, critical situations are detected and a warning (e-mail) is sent to the administrator, in order to take preventive actions. When one of the parameters in Table 3 reaches its maximum value, it is considered a critical situation, which triggers a warning (e-mail). This measure is justified due to the high dependence that machines have of all three parameters of Table 3 to complete successfully its tasks. In other words, if any of these parameters is overly consumed, the respective machine will have a poor performance.

In the case of AIDA's agents, the prevention system is based on the agent's activity frequency. This performance indicator is the interval of time that an agent takes to refresh its log file with its newest activity, in other words it is the frequency that the agent is executed. The Table 4 presents the score table created to forecast agents' faults also based on MEWS. The negative side was removed (as in the model related to the machines) and once it is used one variable, it was added the score four in order to the system becomes more accurate. As Table 4 indicates, the quantifying of the performance indicator is based on percentiles. Inspired in 95-percentile method [13], it was possible create this model. The usage of percentiles demands the necessity to have a large set of information about the indicator. So, during a reasonable period of time, data about agents' activity frequency was collected. In this way it is possible to evaluate the normal behaviour of agents and start the forecasting process.

Table 4. Score table for the agents based on its activity frequency.

\begin{tabular}{|l|c|c|c|c|c|}
\hline Score & $\mathbf{0}$ & $\mathbf{1}$ & $\mathbf{2}$ & $\mathbf{3}$ & $\mathbf{4}$ \\
\hline Activity frequency $(\min )$ & $\leq p 85$ & ]$p 85, p 90]$ & {$[p 90, p 95]$} & $\mid] p 95, p 97,5]$ & $>p 97,5$ \\
\hline
\end{tabular}

If the score is four, it is considered an abnormal situation because the agent does not log its activity for a long time, which is unusual. An e-mail is sent to a system administrator, in order to take speedy action to restore the normal working flow of AIDA. In this way, the administrator prevents serious damages that might occur. This system constantly calculates new percentiles for each agent, improving the system's efficacy to detect abnormal situations.

\section{Results}

The BMaPI was implemented in a machine of the AIDA, which is implemented in CHP. The results presented are related to agent 35, responsible for validating 
laboratory data (e.g. clinical analysis) during the period 10 to 16 of September 2013.

After some time of execution, in this case about 6 days, the BMaPI user can already draw some conclusions about the agent through its properties page shown in figure 1 . Beyond its individual properties and the way it performs its activity (every 1 minute), this page provides a general analysis of the agent activity. It presents the date and the time of the last run of the agent, and its duration in seconds, it shows the user that the activity started at 11 hours and 51 minutes of September 16 had a duration of 10 seconds. The Figure 1 also

\begin{tabular}{|l|l|}
\hline Properties Scheduling & \\
Agent Properties & 35 \\
\hline Code & $35 @$ hsa-aida04 \\
\hline Name & Active \\
\hline State & C: Vhgsalvb $\backslash$ SILAg35.exe \\
\hline Executable & \\
\hline System Properties & hsa-aida04 \\
\hline Machine & 172.21 .201 .22 \\
\hline Adress & \\
\hline Activity Properties & $\$$ every 1 minute \\
\hline Scheduling & Activity Scheduled on: \\
\hline Scheduling Date & $10 / 09 / 201315: 36: 42$ \\
\hline Activity Analysis & \\
\hline Last execution & $16-09-2013$ 11:51:21 \\
\hline Duration of the last activity & $10 \mathrm{~s}$ \\
\hline Number of executions & 8415 \\
\hline Average duration of activities executed & $6 \mathrm{~s}$ \\
\hline Number of errors occurred & 0 \\
\hline Most common error & -- \\
\hline
\end{tabular}

Fig. 1. BMaPI (Properties page from the agent 35).

shows the number of total runs that the agent had since its creation to the time of analysis and the average duration of these activities (6 seconds), this average duration was calculated using the 50th percentile in order to present a measure of central tendency, eliminated so some outlines. With these two data, besides the user can verify its values, may also calculate the number of times the agent has performed its activity for a day, i.e. if the agent is performed 8415 times its activity for nearly 6 days, per day performed approximately 1402 times and by hour 58 times.

To finish, this page makes possible even check the number of errors that occurred along the 8415 activities performed by the agent 35 and also what is the most common error.

The BMaPI also allows the user to control the agent's activity in real time, i.e. the monitoring of activities in a dynamic manner. The monitoring page then has two types of graphs, one graph for the duration of the activities and another for the number of activities performed. By Selecting the type of graph and the 
day for which the user wants to check the agent's activity, comes a set of three groups. The first refers to a daily analysis, followed by a weekly analysis and finally an analysis monthly.

For preventing systems, it was analysed the warning e-mails sent by both systems presented in the Section 4.2.

The preventing system for machines, based on Table 3, detected 6 critical situations during the period between 30th July to 25th September 2013. These 6 occurrences happened in three machines of AIDA (2 occurrences per machine). For example, the e-mail received on 30th July reported that a machine was consuming $97 \%$ of CPU and $91 \%$ of memory. The administrators were properly informed and preventive actions were taken, avoiding serious damages in AIDA's functioning. Similar situations have happened to the other five occurrences.

In the case of agents' forecasting system based on the Table 4, three maintenance situations were realized in different days during the tests phase (June and July 2013). The process of maintenance, naturally, caused a stop in several agents' activities. In all of these three situations, the agents' fault forecasting system detected that those agents stopped. This fact proves that the system is capable of quickly detect an agents' irregular situation preventing bigger damages. During the same period, two critical situations (score 4) were detected. In both situations the agents were stopped for more than 15 minutes and they usually register their activities each minute. These occurrences were successfully reported to administrators who treated the case.

Based on the results presented, it can be stated that the developed systems contribute to the improvement of AIDA's integrity and high availability and its functionalities and usability are increased through the BMaPI.

\section{Conclusions and Future Work}

After a detailed study about AIDA platform, it arises the need to eliminate some of its weaknesses. Thus were developed and implemented monitoring and prevention of failures systems, in order to improve not only the functionality and usability of the platform but also give it a greater availability.

Regarding the prediction of failures, it was possible to adapt the model MEWS to the AIDA's machines and agents' performance, to build tools with a high potential to assist system administrators and consequently improve the quality of services provided.The results show that the BMaPI platform allows the user to make a detailed analysis about the activity of each agent.

It is proposed as future work the relation between BMaPI and the machine monitoring system, in order to make a proper balancing of resources automatically. Namely the control of machines' CPU and memory so that whenever one machine reach a certain threshold the agents that are hosted on this machine can migrate to another machine. This way it is possible a managing the resources in an automatic and efficient way. Another future work related to the fault forecasting systems developed is the improvement of their alert module, adding alerts via SMS in extreme cases. 


\section{Acknowledgement}

This work is financed with the support of the Portuguese Foundation for Science and Technology (FCT), with the grant SFRH/BD/70549/2010 and within project PEst-OE/EEI/UI0752/2011.

\section{References}

1. Duarte, J., Salazar, M., Quintas, C., Santos, M., Neves, J., Abelha, A., Machado, J.: Data quality evaluation of electronic health records in the hospital admission process. In: Computer and Information Science (ICIS), 2010 IEEE/ACIS 9th International Conference on. (aug. 2010) $201-206$

2. Weber-Jahnke, J., Peyton, L., Topaloglou, T.: ehealth system interoperability. Information Systems Frontiers 14(1) (2012) 1-3

3. Miranda, M., Duarte, J., Abelha, A., Machado, J., Neves, J.: Interoperability in healthcare. In: EUROPEAN SIMULATION AND MODELLING CONFERENCE (ESM 2010), Hasselt, Belgium, 2010 - "Proceedings of the European Simulation and Modelling Conference (ESM 2010)". [S.1.]. (2010)

4. Rogers, R., Peres, Y., Müller, W.: Living longer independently-a healthcare interoperability perspective. e \& i Elektrotechnik und Informationstechnik 127(7) (2010) 206-211

5. Abelha, A., Machado, J., Santos, M., Allegro, S., Rua, F., Paiva, M., Neves, J.: Agency for integration, diffusion and archive of medical information. In: Proceedings of the Second IASTED International Conference-Artificial Intelligence and Applications, Benalmadena, Spain. (2002)

6. Portela, C.F., Santos, M.F., Silva, Á., Machado, J., Abelha, A.: Enabling a pervasive approach for intelligent decision support in critical health care. In: ENTERprise Information Systems. Springer (2011) 233-243

7. Devaney, G., Lead, W.: Guideline for the use of the modified early warning score (MEWS). Technical report, Outer North East London Community Services (2011)

8. Albino, A., Jacinto, V.: Implementação da escala de alerta precoce - EWS. Technical report, Centro Hospitalar do Barlavento Algarvio, EPE, Portimão (2010)

9. Silva, P., Quintas, C., Duarte, J., Santos, M., Neves, J., Abelha, A., Machado, J.: Hospital database workload and fault forecasting. In: 2012 IEEE EMBS Conference on Biomedical Engineering and Sciences. (Malaysia, 2012)

10. Boudec, J.: Performance Evaluation of Computer and Communication Systems. Computer and communication sciences. EPFL Press (2010)

11. Boshier, A.: Windows Management Instrumentation: A Simple, Powerful Tool for Scripting Windows Management [J]. MSDN Magazine 4 (2000)

12. Tereso, M., Bernardino, J.: Open source business intelligence tools for smes. In: Information Systems and Technologies (CISTI), 2011 6th Iberian Conference on, IEEE (2011) 1-4

13. Dimitropoulos, X., Hurley, P., Kind, A., Stoecklin, M.P.: On the 95-percentile billing method. In: Passive and Active Network Measurement. Springer (2009) $207-216$ 